\title{
Bifid Uvula: Tip of an Ice Berg
}

\author{
Jyoti Sharma, Neetu Khanduri, Monica Chhikara, Chhavi Wadhwa
}

Department of Anaesthesiology, Pandit Bhagwat Dayal Sharma Post Graduate Institute of Medical Sciences, Affiliated to University of Health Sciences, Rohtak, Haryana - 124001, India.

\section{Corresponding Author:}

Dr. Jyoti Sharma

Email id: doctorjyotisharma@yahoo.in

This is an Open Access article distributed under the terms of the Creative Commons Attribution License (creativecommons.org/ licenses/by/3.0).

Received : July 15, 2019

Accepted : August 26, 2019

Published : September 20, 2019

\begin{abstract}
Background: A bifid uvula is an abnormal split or division in the uvula or tissue that hangs down at the end of soft palate in the roof of mouth. It has lesser amount of muscular tissues as compared to normal uvula. It is often noticed in infancy and rarely found in adults. Sometimes a bifid uvula is an indication of a submucous cleft palate. Submucous cleft palate can lead to problems with speech, swallowing and may be associated with ear problems. In majority of cases genetic association is present in patients with bifid uvula. Case Report: A 26 year old female posted for surgery for thyroglossal cyst was found to have bifid uvula during pre-anaesthetic check-up. She underwent surgery under general anaesthesia uneventfully. Conclusion: Detailed pre-anaesthetic workup and continuous peri-operative vigilance is required in cases of bifid uvula.
\end{abstract}

Keywords: Cleft Palate, Face, General Anesthesia, Speech, Uvula.

\section{Introduction}

A bifid uvula is an abnormal split or division in the uvula or tissue that hangs down at the end of soft palate in the roof of mouth [1]. Estimated prevalence is 1:1250-1:5000. Bifid uvula can be found in $0.44 \%-3.3 \%$ of normal individuals [2]. Patients with bifid uvula can also present with cerebral aneurysm, aortic rupture or dissection [3]. Bifid uvula can be a manifestation of a rare disorder known as Cornelia de Lange syndrome. It is a genetic disorder which can lead to severe physical and intellectual anomalies [4,5]. Other syndromes associated with bifid uvula are RitscheSchinzel syndrome, Loeys Dietz syndrome, Marfan syndrome and Stickler syndrome [6,7].

\section{Case Report}

A 26 year old female weighing $40 \mathrm{~kg}$, presented for pre-anesthetic checkup for fitness for thyroid surgery for thyroglossal cyst. She belonged to ASA grade 1 . There was no history of difficulty in swallowing and breathing, voice change, pain abdomen or syncopal attack. She had a history of surgery for defect in ear lobule in childhood under general anaesthesia. There was no significant family history present. Airway examination was normal except for bifid uvula. Her blood reports, ECG and chest X-ray were within normal range. No other clinical features were suggestive of any syndrome. She was advised abdominal ultrasound, brain imaging and echocardiography which was within normal range.

She was pre-medicated with tab ranitidine $150 \mathrm{mg}$ and tab alprazolam $0.25 \mathrm{mg}$ one night prior and early morning on the day of surgery. Intravenous line was secured with $18 \mathrm{G}$ cannula. She was induced with inj. glycopyrrolate $0.2 \mathrm{mg}$, inj. fentanyl 80 microgram and propofol $80 \mathrm{mg}$ and inj. vecuronium $5 \mathrm{mg}$. Gentle laryngoscopy was done to avoid contact with hard and soft palate and trachea was intubated with tube size $7.0 \mathrm{~mm}$. Vitals just after intubation were $H R=86 / \mathrm{min}$, 
$\mathrm{NIBP}=130 / 80 \mathrm{mmHg}$ and $\mathrm{SPO}_{2}=100 \%$. Anesthesia was maintained with oxygen, nitrous oxide and sevoflurane and inj. vecuronium $1 \mathrm{mg}$. Her surgery was uneventful and she was extubated without any complication and was shifted to recovery room.

\section{Discussion}

The term bifid uvula means the partial or full bifurcation of the uvula. Submucous cleft palate shows malpositioning of the palate muscles and may result in velopharyngeal insufficiency and hypernasality. Samanta et al. in 2013 reported a case of 16 year old who had bifid uvula and mild hypertelorism in pre-anaesthetic check up. Other than this no other abnormal finding was present in the patient. Patient underwent retinal surgery. Intraoperative hemodynamics remained within normal range. Patient developed T-wave inversion in lead II. Esmolol $30 \mathrm{mg}$ was used to prevent extubation response. Patient could not be extubated as his GCS did not improve. He had anisocoria. He was shifted to ICU and CT was performed. Ruptured aneurysm in the right middle artery was found. Immediately nimodipine was started. Patient was then shifted to neurosurgery theatre and craniotomy and clipping was performed. Patient was then shifted to ICU and was discharged to home later. He suggested that bifid uvula may be a warning sign of the syndrome with internal anatomical or functional changes without any external manifestation. In the present study also we did all the investigations to rule out any anatomical variation [1]

Khasbage SD in 2017 reported bifid uvula in three members of a family. In the same family he reported three cases of bifid uvula and palatal cleft. Fifty eight year old male presented with the complaint of toothache, he had bifid uvula and palatal perforation. His elder son of age 28 years presented with the complaint of decay tooth, he also had bifid uvula and palatal perforation. His younger son of age 26 years presented with the complaint of discoloured teeth, he had bifid uvula and submucosal cleft. They could not assess

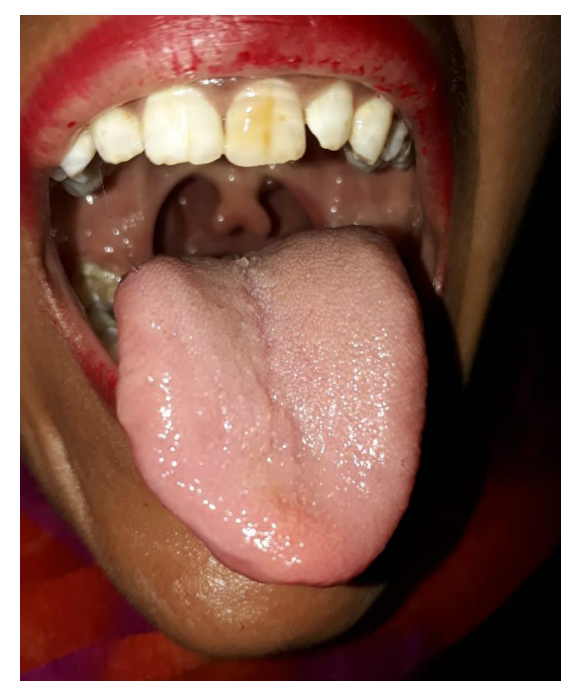

Fig.1: Bifid uvula.

the patients further as patients were not willing. Khasbage SD suggested that an anaesthetist must ask for detailed family and genetic history, clinical relevant investigations and specialty consultation [4]. In contrast to their case report we conducted all the relevant investigations which were in normal range. Vilacosta I reportd a case of bifid uvula associated with aortic aneurysm in 2008. The patient had a family history of aortic disease. In physical examination patient had proptosis, retrognathia, and bifid uvula. He was diagnosed with Loeys-Dietz syndrome type 1. Surgery for aortic root replacement was performed which was successful [3].

Airway management may not be challenging in all the cases with bifid uvula but all the patients must be looked for any anatomical variation and features of associated syndromes.

\section{Conclusion}

The detailed workup of the patient with bifid uvula should be done during pre-anaesthetic evaluation to rule out any anatomical or physiological variation. Detailed history of the patient and the family should be taken to rule out any genetic alteration. Thorough general examination should be done to rule out syndromes associated with 
bifid uvula. Abdominal and brain imaging should be done to rule out any aneurysm. Anesthetist should be prepared for all the complications intraoperatively. Gentle laryngoscopy should be done as submucosal cleft can be present. We finally recommend a constant vigilance of the patient throughout the peri-operative period.

Contributors: JS participated in the patient care, and manuscript writing; $\mathrm{NK}$ and $\mathrm{MC}$ participated in study conception and writing of the paper; $\mathrm{CW}$ participated in revising the manuscript critically. JS will act as a study guarantor. All authors approved the final version of this manuscript.

Funding: None; Competing interests: None stated.

\section{References}

1. Samanta S, Samanta S. Bifid uvula: Anaesthetists don't take it lightly. Saudi J Anaesth. 2013;7:482-484.

2. Sales SAG, Santos ML, Machado RA, Dias VO,
Nascimento JE, Swerts MSO, et al. Incidence of bifid uvula and its relationship to submucous cleft palate and a family history of oral cleft in the Brazilian population. Braz J Otorhinolaryngiol. 2018;84:687-690.

3. Vilacosta I, Godoy VC. Bifid uvula and aortic aneurysm. N Eng J Med. 2008;359(2):e2.

4. Khasbage SD. Bifid uvula in three family members of a family. Int J Oral Craniofac Sci. 2017;3:17-19.

5. Callea M, Montanari M. Bifid uvula and submucous cleft palate in cornelia de lange syndrome. J Int Dent Med Res. 2011;4:74.

6. Konya MN, Elmas M, Erginoglu SE, Yasil M. A rare case of 3c disease: Ritscher-Schinzel syndrome presenting with recurrent talipes equinovarus. Int J Surg case Rep. 2015;7:130-133.

7. Lituania M, Tonni G. Bifid uvula and familial Stickler syndrome diagnosed prenatally before the sonographic "equals sign" landmark. Arch Gynecol Obstet. 2013;288:483-487. 